\title{
¿Son reales los beneficios de la arquitectura empresarial? Un análisis desde las capacidades organizacionales
}

\section{Are the benefits of enterprise architecture real? An analysis from organizational capacities}

Maykel Manuel Chávez Rodríguez

Empresa de Telecomunicaciones de Cuba S.A.

maykel.chavez@etecsa.cu
Especialista de Calidad de la Empresa de Telecomunicaciones de Cuba S.A., Carretera a Maleza, No 39, entre Tercera y Ouinta, Reparto Camacho, Santa Clara, Villa Clara, CUBA.

INFORMACIÓN ARTÍCULO

Recibido: 9 de Enero 2021

Aceptado: 1 de Junio 2021

\section{Resumen \\ Propósito: Analizar los beneficios de la arquitectura empresarial utilizando las capacidades organizacionales. \\ Diseño/Metodología: La investigación es cualitativa con enfoque exploratorio y se utilizan métodos y técnicas de análisis documental, como el análisis de contenidos y la descripción bibliográfica a través de la identificación, indexación, revisión y resumen. \\ Resultados: Se demostró que los trabajos e iniciativas de evaluación de los beneficios de arquitectura empresarial analizados, carecen de una clasificación clara y no ofrecen evidencias empiricas. Sobre esta base se establece el análisis de los beneficios de la arquitectura empresarial a través de cuatro capacidades organizacionales. \\ Implicaciones prácticas: Utilizar las capacidades organizacionales como elementos mediadores entre las iniciativas de arquitectura empresarial y los resultados de las organizaciones, sirviendo de vínculo para aportar pruebas empíricas. \\ Originalidad/Valor: La investigación aborda el tema de los beneficios de la arquitectura a través de un enfoque novedoso que abre nuevas posibilidades para estudios empíricos sobre la temática.}

\begin{abstract}
Purpose: Analyze the benefits of enterprise architecture using organizational capabilities.

Design / Methodology: The research is qualitative with an exploratory approach and documentary analysis methods and techniques are used, such as content analysis and bibliographic description through identification, indexing, review and abstract.

Results: Previous studies and initiatives assessing the benefits of enterprise architecture showed undetermined classification and lack of empirical evidence. Based on this, the analysis of the benefits of business architecture is established through four organizational capacities.

Practical implications: Use organizational capabilities as mediators among enterprise architecture initiatives and organizational results as a link to provide empirical evidence.

Originality / Value: The research addresses the issue of the benefits of architecture through a novel approach that opens up new possibilities for further studies.
\end{abstract}

\section{Palabras Claves:}

Arquitectura empresarial

Beneficios

Capacidades

Capacidades organizacionales
ARTICLE INFO

Received: 9 January 2021

Accepted: 1 June 2021
Keywords:

Business architecture Benefits

Capabilities

Organizational capabilities 


\section{INTRODUCCIÓN}

Desde que J.A. Zachman publicó su famoso artículo en la "Revista de Sistemas de IBM" en 1987 (Zachman, 1987), la arquitectura empresarial no ha parado de generar interés tanto en el ámbito académico como empresarial (Minoli, 2008; Sessions y deVadoss, 2014). El mundo es cada vez más digital y la tecnología ya no es un lujo de las grandes corporaciones, es un requisito para cualquier industria (Jayakrishnan y cols., 2019). No existe acuerdo sobre el momento en que surge exactamente la arquitectura empresarial. Según los trabajos de AlDhaheri (2011); Roach (2011), Sessions y deVadoss (2014) y Kotusev (2016), J.A. Zachman marca el punto de partida con su reconocido artículo y por esta razón ha sido considerado por muchos como "el padre" de la arquitectura empresarial. Otros autores como Farazmand y Moeini (2011), Cretu (2014), Orabona (2016) y Marini (2019), reconocen que es indiscutible el aporte de Zachman, pero aseguran que la arquitectura empresarial nace mucho antes, después de un proceso evolutivo desde la arquitectura de los sistemas de información. A pesar de la divergencia de criterios, no existe duda de que la arquitectura empresarial tiene una alta repercusión en la contextualización del nuevo paradigma de organización que debe ser innovadora, flexible e interconectada.

Los beneficios de la arquitectura empresarial han sido reseñados en muchas publicaciones y trabajos de investigación. La forma en que gestiona la complejidad (Sessions, 2011), la forma en que facilita la comprensión holística de la organización (Bernard, 2012; Lauvrak, Michaelsen y Olsen, 2017) y la capacidad de integración de la tecnología con el negocio (Theuerkorn, 2005; Abdullah, 2017; Niemi y Pekkola, 2019) son algunos de los beneficios más señalados. A pesar de que la existencia de evidencias sobre la utilidad y beneficios de la arquitectura empresarial ha sido reseñada en varios trabajos y publicaciones, esta no ha estado exenta a las críticas y cuestionamientos. La mayoría de los señalamientos se relacionan con la poca evidencia de su impacto real y muchos de sus detractores plantean que es difícil encontrar ejemplos de arquitecturas desarrolladas con éxito en la práctica (Banaeianjahromi y Smolander, 2017; Banaeianjahromi, 2018). La utilización de marcos de trabajo obsoletos (Rouhani y $\mathrm{Ni}$ kpay, 2012), la poca uniformidad de los proyectos (Louis y Lapalme, 2018) y su poca flexibilidad
(Hylving y Bygstad, 2018), son de los aspectos más frecuentes en los fracasos en la práctica de la arquitectura empresarial. Para Noran (2018) y Kotusev (2018) existen cuestionamientos suficientes de la utilidad práctica de la arquitectura empresarial, muchas de las iniciativas no satisfacen todas las dimensiones importantes de la empresa y con frecuencia pueden entrar en conflicto con las estrategias comerciales (Kotusev, Kurnia, Dilnutt y Taylor 2020). A todo esto, se le suma la falta de cooperación entre profesionales que practican la disciplina (Pähler y cols., 2019) y la poca información disponible sobre la evaluación de la calidad y efectividad de las arquitecturas (Rogier, Wetering y Joosten, 2020; Niemi y Pekkola, 2019)

Se ha demostrado que en la mayoría de los fracasos de proyectos o iniciativas de arquitectura empresarial son responsables las organizaciones que la aplican y no la disciplina en sí misma. Considerar la arquitectura empresarial como un fin y no como un medio (Hartmann, 2008), el no establecimiento de una metodología estándar (Saha, 2012) y la falta de capacidad para demostrar que es una fuente de beneficios (Niemi y Pekkola, 2016) son algunos de los errores que más se cometen. Es en esta última problemática en la que se basa el presente trabajo. Según Niemi y Pekkola (2016), en su trabajo crítico sobre la utilidad de la arquitectura empresarial, no es que falten estudios o análisis de los beneficios de la arquitectura empresarial, es que la mayoría de estos no se conducen a través de evidencias validadas en la práctica (Niemi y Pekkola, 2016). Según estos autores estas evidencias son difíciles de obtener debido a que los resultados de las organizaciones involucradas son difíciles de correlacionar con la arquitectura aplicada, al menos de forma directa. Muchos autores han buscado contribuir a su consolidación y resolver el eterno dilema de los detractores de la disciplina que la han llegado a llamar "el nuevo tema de moda". Autores como Kotusev, Kurnia, Dilnutt y Taylor (2020) han sido muy incisivos en mostrar la parte no expuesta de la arquitectura empresarial como un tema del momento, dado que ha sido difícil demostrar la influencia directa de la arquitectura empresarial sobre los resultados se han utilizado una amplia variedad de variables relacionadas tanto financieras como operacionales (Kotusev y cols., 2020). Las investigaciones realizadas, muchas de ellas se abordan en este artículo, no han satisfecho las expectativas y los detractores de la arquitectura empresarial siguen dando de qué hablar (Kotu- 
sev y cols., 2020). Para establecer nuevas pautas sobre la temática, en este trabajo se propone un nuevo enfoque en el análisis de los beneficios que se basan en las capacidades organizacionales como elementos mediadores entre las iniciativas de arquitectura empresarial y sus resultados en la práctica.

En esta investigación los beneficios de la arquitectura empresarial se tratan desde una perspectiva diferente a los enfoques tradicionales. Se incluyen cuatro capacidades organizacionales como variables mediadoras entre la arquitectura empresarial y los resultados de las organizaciones que la aplican: Capacidad de integración de tecnologías de la información, capacidad de alineación estratégica, capacidad de gestión de la complejidad y capacidad de innovación. A través de estas se establece a nivel teórico se un necesario vínculo para la evaluación del impacto real de la arquitectura empresarial. Además, se aporta un enfoque donde los investigadores y especialistas pueden a través de casos de uso demostrar la pertinencia de la arquitectura empresarial de una forma objetiva y comprobable. Las capacidades organizacionales son elementos que pueden correlacionarse con los resultados de las organizaciones y sus procesos de forma objetiva. El análisis de los beneficios de la arquitectura empresarial enfocándolos a las capacidades organizacionales aporta mayor visibilidad y veracidad a los argumentos para llegar a convencer a aquellos, académicos o no, que no creen en su carácter innovador y revolucionario de las ciencias empresariales en general.

\section{MÉTODO}

La revisión de la literatura constituye una de las etapas fundamentales de toda investigación (Sureda, Oliver, Comas y Guerrero, 2010; Baena, 2017). Para Hernández, Fernández y Baptista (2014), la investigación documental es utilizada para mediante la identificación, consulta y análisis de bibliografía y otros materiales con independencia de su soporte investigar una realidad o fenómeno dado. En la presente investigación los tipos de fuentes donde se extraen, analizan y se indexan documentos son tanto primarias como secundarias (Morán y Alvarado, 2010). Las fuentes primarias fueron libros (electrónicos en su gran mayoría), publicaciones periódicas, informes científicos y técnicos, actas de congresos o simposios, tesis (maestría y doctorado), monografías y artículos académicos. Por su parte, las fuentes secundarias fueron boletines o revistas de resúmenes bibliográficos, catálogos que contienen descripciones y reseñas bibliográficas.

Los métodos y técnicas utilizadas en la investigación son los de análisis de contenido. Estos métodos utilizan la lectura (textual o visual) como instrumento de extracción de información a través del método científico (Díaz, 2018) y tienen una metodología perfectamente establecida (Guix, 2008). Se seleccionan los documentos y materiales de la arquitectura empresarial de acuerdo al enfoque con que se trata el tema de sus beneficios e impacto en las organizaciones. Se realiza la descripción física para la identificación inequívoca del documento y el análisis de los argumentos para la indexación, resumen y clasificación de temas a través de las técnicas siguientes:

Análisis formal externo (descripción bibliográfica): Actúa sobre el soporte e identifica los datos externos de un documento que lo distinguen de otro, proporcionando una identificación individual. Se laboran fichas bibliográficas y fichas de trabajo (Morán y Alvarado, 2010).

Análisis formal interno (análisis de contenidos): Opera sobre el contenido temático del documento y logra una representación de la información tratada. Se realiza la indización a través de descriptores. (Morán y Alvarado, 2010).

Debido a evidentes razones de espacio no es posible abordar en este trabajo la totalidad de estudios e investigaciones que tratan el tema de los beneficios de la arquitectura empresarial. Los criterios utilizados para la selección de publicaciones se describen a continuación:

Pertinencia: La publicación o material consultado debe relacionarse estrictamente con la utilidad y los beneficios de la arquitectura empresarial.

Autenticidad: Se toman solo aquellas publicaciones que provienen de revistas indexadas, de centros de estudios de alto impacto o de sitios web de instituciones que trabajan el tema de la arquitectura empresarial formalmente.

Representación y significado: Se seleccionan aquellos trabajos donde el análisis de los beneficios se realice teniendo en cuenta un enfoque claro, con independencia de la naturaleza de este. 
Actualidad: Implica que los textos deben ser lo suficientemente actuales y representen los últimos avances teóricos o los antecedentes empíricos más recientes. En el caso de la presente investigación se tomaron desde el año 2000 hasta 2019, tomando en cuenta que en los inicios del siglo XXI es donde coinciden la mayor cantidad de autores en que es el comienzo del auge de la arquitectura empresarial tal y como la conocemos hoy.

\section{DESARROLLO DEL TEMA}

\section{Arquitectura empresarial.}

\section{Conceptos fundamentales}

Existe una gran cantidad de interpretaciones del concepto de arquitectura empresarial (Nilsen, 2012; Kotusev, Singh y Storey, 2015; Ferreira de Santana, 2017; Abdallah y Abran, 2019; Pähler y cols., 2019; Marini, 2019; Louis y Lapalme, 2019; Ajer, 2020; Kotusev, Kurnia, Dilnutt y Taylor, 2020 y Rogier, Wetering y Joosten, 2020) y no existe aún una definición definitiva (Niemann, 2005; Ross, Weill y Robertson, 2006; Winter y Fischer, 2007; Sidorova y Kappelman, 2010; Perroud y Inversini, 2013; Kotusev y cols., 2015; Nygård y Olsen, 2016 y Dang y Pekkola, 2017) a pesar de que se superponen muchos de los conceptos. En esta variedad se destacan varios aspectos comunes como es el caso de que la arquitectura empresarial puede considerarse una presentación holística de la organización, una metodología detallada de migración a un estado deseado o una lógica de organización. Aunque existe gran variedad en cuanto a conceptos, está muy clara la definición de la arquitectura empresarial como elemento integrador de elementos como la estrategia, las personas, los negocios, los datos y las tecnologías de la información (Moscoso, Paredes y Luján, 2019 y Abunadi, 2019). A pesar de que la línea central de todas las definiciones es común, la dispersión conceptual se da en aspectos como el objetivo de las arquitecturas, así como su papel en la estructuración de las organizaciones. Según Louis y Lapalme (2018) la dispersión conceptual es una muestra de que la arquitectura empresarial no ha alcanzado su máximo nivel de madurez.

La definición de arquitectura empresarial ha sido muy enfocada en la comparación con el significado del término en el ámbito de la construc- ción (Vermeulen 2013; Perroud y Inversini 2013 y Ferreira 2017), ya que la palabra "arquitectura" tiene que ver con el diseño de estructuras (Vermeulen, 2013). Al llevar esta analogía al plano empresarial el término adquiere una nueva connotación ya que se enfoca al diseño o plano de una organización u empresa (Marini 2019). Inicialmente se consideró el término vinculado con la noción de la arquitectura de sistemas de información (Orabona, 2016; Kotusev, 2016 y Marini 2019), pero hoy su alcance es mucho más amplio (Simon, Fischbach y Schoder, 2013), ya que es capaz de describir una arquitectura de referencia o habilitar la descripción de esta (Tome y Costa, 2010). En la amplia gama de definiciones de arquitectura empresarial resaltan algunos aspectos comunes (Vermeulen, 2013) como es el caso de su enfoque holístico, la relación del negocio con las tecnologías de la información y el establecimiento de un plan detallado de migración de un estado actual a un estado futuro.

En cuanto a su enfoque holístico la arquitectura empresarial puede considerarse como una representación integral de la organización (Fischer y cols., 2010; Winter y Aier, 2010; Aier, Fischer y Winter, 2011; Nygård y Olsen 2016; Qurratuaini, 2017; Armour y Kaisler, 2018; Werewka, 2018; Hylving y Bygstad 2018; Gampfer, 2019; Fratila, 2020; Ajer, 2020 y Lê, 2020). Este enfoque es una de las características clave de la arquitectura empresarial (Lankhorst, 2009), porque se logra una visión sistémica (Vermeulen 2013; Perroud y Inversini 2013; Kar y Thakurta, 2018 y Erdenebold, Rho y Hwang, 2019) y facilita la gestión de la complejidad (Niemann 2005; Sessions 2008 y Niemi 2016). La arquitectura empresarial se considera como un grupo de descripciones lógicas que se vinculan entre sí (Winter y cols. 2010; Fischer, Winter y cols., 2010).

En cuanto a la relación entre el negocio y las tecnologías, la arquitectura empresarial es la lógica de organización de los procesos de negocio (Kamoun, 2013 y Ross, Weill y Robertson, 2014) y su infraestructura tecnológica (Lankhorst 2009; Aier y Schelp, 2010; Bernard 2012; Niemi y Pekkola, 2013; Abunadi 2019 y Sukur, 2020). Esta se apoya en la armonización (Azizi y Sumitra, 2019), la integración (Randall, 2018; Priyanto, 2013) y la estandarización de los requisitos operacionales (Ross y cols. 2014). Un factor clave de la arquitectura empresarial es la alineación negocio-tecnología (Tamm, Seddon, Shanks, Reynolds y Frampton, 2011; Niemi y Pekkola, 
2013; Niemi 2016 Okhrimenko, 2017; Banaeianjahromi y Smolander 2017). Se considera además una herramienta necesaria para la gestión de la efectividad empresarial (Lankhorst 2009), que considera la eficiencia en el uso de la tecnología disponible (Lukhele, Ngassam y Osunmakinde, 2015; Sultanow, Schroeder, Schroeder y Cox, 2016; Armour y Kaisler, 2018 y Caruso, 2019) y ayuda a la orientación de las inversiones tecnológicas (Banaeianjahromi 2018 y Sari, Rahmawati y Harafani, 2019).

Considerando el plan detallado de migración la arquitectura empresarial se define como un plan maestro (Schekkerman, 2011; Perroud y Inversini 2013 y The Open Group, 2018), que contiene una descripción del estado actual de una organización (Kotusev, 2019), un plan de transición y la descripción de un estado futuro (Open Group, 2011; Tamm, Seddon, Shanks, Reynolds y Frampton, 2011; Lange, Mendling y Recker, 2012; Bernard 2012). Es soporte para las organizaciones en un contexto dinámico (Kotusev 2019), al establecer bases de pensamiento para tomar decisiones (Sofyana y Putera, 2019) y la implementación de la estrategia (Chakraborty y Sarkar, 2017 y Erdenebold, Rho y Hwang, 2019). Para Kotusev (2019), la arquitectura empresarial incluye una arquitectura base, una arquitectura destino y un plan de secuencia para llegar a ella. Actúa modelando elementos de las organizaciones, aportando capacidades a la empresa e influyendo en su comportamiento y eficiencia (Giachetti, 2015).

\section{Revisión de métodos y estudios de los beneficios de la arquitectura empresarial. Aciertos y des- aciertos.}

Los beneficios de la arquitectura empresarial no tardaron mucho en ser estudiados desde los primeros años del surgimiento de la disciplina. Las primeras contribuciones se originaron en la comunidad académica e inicialmente no tenían un basamento empírico significativo debido a una superposición entre los beneficios identificados (Tamm, Seddon, Shanks, Reynolds y Frampton, 2011). Los beneficios de la arquitectura empresarial han sido objeto de varias investigaciones académicas (Plessius, Steenbergen y Slot, 2013), pero no hay una forma común de clasificarlos (Niemi, 2016). En la tabla 1 se muestra una reseña de los trabajos y publicaciones consultados en la presente investigación.
De la gran variedad de estudios mencionados en la tabla 1 resaltan algunos por lo interesante de sus propuestas. El "Modelo de Beneficios de Arquitectura Empresarial" (EABM por sus siglas en inglés) estipula que se puede agregar valor a las organizaciones a través de cuatro habilitadores de beneficios: alineación organizacional, disponibilidad de información, optimización de la cartera de recursos y la complementariedad de los recursos (Proper, Lankhorst, Schönherr. Barjis y Overbeek, 2012; Kamoun, 2013). Algunos autores usan marcos de rendimiento existentes en otros campos de las ciencias empresariales. Un ejemplo particular es el "Cuadro de Mando Integral", que ha sido muy utilizado para clasificar los beneficios de la arquitectura empresarial (Plessius, Steenbergen y Slot, 2013). En el estudio de Baharudin Jusuf y Kurnia (2017) se establecen dos fases de análisis-síntesis de literatura crítica y se realizan entrevistas cualitativas con expertos para validar la lista de beneficios e impulsores. Esta propuesta tiene puntos en común con un análisis precedente de Lange y cols. (2012) que utilizan también estas dos fases, pero a través de un estudio bibliográfico más abarcador.

Existen otros autores como Rodríguez y Amaral (2010) que abordan la cuestión del valor utilizando un enfoque multidimensional. Estos autores asumieron el reto de la evaluación del impacto financiero de la arquitectura empresarial definiendo cuatro valores fundamentales: valor estratégico, valor operativo, valor del usuario y valor económico (Rodríguez y Amaral, 2010). Este estudio es coherente con la línea del análisis de valor de autores como Gayatri y Nagalakshmi (2019) que abordan la evaluación a través de varios promotores de valor. Los estudios de los beneficios basados en el valor carecen todos de un análisis objetivo de como la arquitectura empresarial es capaz de generar ese valor de forma directa. Según la propuesta de Niemi y Pekkola (2019) existen beneficios cuantificables y no cuantificables, en el caso de estos últimos pueden clasificarse en duros e intangibles. A pesar de la existencia de las iniciativas abordadas en la tabla 1 y sus resultados, se evidencia la prevalencia de lo teórico sobre lo empírico. La identificación de los beneficios cuantificables sigue siendo una asignatura pendiente cuando se trata de demostrar la verdadera utilidad de la arquitectura empresarial. 
Tabla 1: Trabajos y publicaciones consultados en la presente investigación

\begin{tabular}{|c|c|c|}
\hline No & Autor & Publicación \\
\hline 1 & (Gabier y cols., 2005) & $\begin{array}{l}\text { Benefits and factors driving enterprise architecture development in a large } \\
\text { South African Utility Company }\end{array}$ \\
\hline 2 & (Kluge y cols., 2006) & How to realise corporate value from enterprise architecture \\
\hline 3 & (Lankhorst y cols., 2006) & Enterprise architecture: Management tool and blueprint for the organisation \\
\hline 4 & (Cane, 2007) & Measuring the impact of enterprise architecture \\
\hline 5 & (Dyer, 2009) & Measuring the benefits of enterprise architecture. \\
\hline 6 & (Bonnet, 2009) & Measuring the effectiveness of enterprise architecture implementation \\
\hline 7 & (Op 't Land y cols., 2009) & Creating value by informed governance \\
\hline 8 & (Liimatainen, 2009) & Evaluating benefits of government enterprise architecture \\
\hline 9 & (Sidorova y Kappelman, 2010) & $\begin{array}{l}\text { Realizing the benefits of enterprise architecture: An actor-network theory } \\
\text { perspective }\end{array}$ \\
\hline 10 & (Lin y Dyck, 2010) & The value of implementing enterprise architecture in organizations \\
\hline 11 & (Lux y cols., 2010) & $\begin{array}{l}\text { Understanding the performance impact of enterprise architecture manage- } \\
\text { ment }\end{array}$ \\
\hline 12 & (Rodriguez y O’Neill, 2010) & Framework based on benefits management and enterprise architecture \\
\hline 13 & (Boucharas y cols., 2010) & $\begin{array}{l}\text { The contribution of enterprise architecture to the Achievement of Organiza- } \\
\text { tional Goals. }\end{array}$ \\
\hline 14 & (Tamm y cols., 2011) & How Does Enterprise Architecture Add Value to Organisations? \\
\hline 15 & ( AlDhaheri, 2011) & $\begin{array}{l}\text { A study on Enterprise Architecture framework effects on business processes } \\
\text { automation }\end{array}$ \\
\hline 16 & (Nassiff, 2012) & Understanding the Value of Enterprise Architecture for Organizations. \\
\hline 17 & (Lange y cols., 2012) & Realizing benefits from enterprise architecture: a measurement model \\
\hline 18 & (Plessius y cols., 2013) & Towards an Enterprise Architecture Benefits Measurement Instrument \\
\hline 19 & (Van Zijl y Van Belle, 2014) & $\begin{array}{l}\text { Organisational Impact of Enterprise Architecture and Business Process Capa- } \\
\text { bility in South African Organisations }\end{array}$ \\
\hline 20 & (Henk y cols., 2014) & Perceived benefits from enterprise architecture \\
\hline 21 & (Lukhele y cols., 2015) & $\begin{array}{l}\text { A Framework for Measuring the Value of Enterprise Architecture in South Af- } \\
\text { rican Telecommunications Organizations }\end{array}$ \\
\hline 22 & (Foorthuis y cols., 2015) & A theory building study of enterprise architecture practices and benefits \\
\hline 23 & (E. Niemi, 2016) & Enterprise Architecture Benefit Realization \\
\hline 24 & (Zaher, 2017) & $\begin{array}{l}\text { The Enterprise Architecture: An Empirical Study on The Organisational Bene- } \\
\text { fits and Success Factors }\end{array}$ \\
\hline 25 & (Baharudin Jusuf y Kurnia, 2017) & Understanding the Benefits and Success Factors of Enterprise Architecture \\
\hline 26 & (Becker y cols., 2017) & $\begin{array}{l}\text { On the Relevance of Enterprise Architecture and IT Governance for Digital } \\
\text { Preservation }\end{array}$ \\
\hline 27 & (Lauvrak, y cols., 2017) & $\begin{array}{l}\text { Benefits and challenges in enterprise architecture management: a case study } \\
\text { of the Norwegian labour and welfare administration }\end{array}$ \\
\hline 28 & (Plessius y cols., 2018) & The enterprise architecture value framework \\
\hline 29 & (Searle, 2018) & The Benefits of Enterprise Architecture for Library Technology Management. \\
\hline 30 & (Gayatri y Nagalakshmi, 2019) & $\begin{array}{l}\text { Benefits of Government Enterprise Architecture: Context of certain EA initia- } \\
\text { tives in India }\end{array}$ \\
\hline 31 & (E. Niemi y Pekkola, 2019) & The Benefits of Enterprise Architecture in Organizational Transformation \\
\hline
\end{tabular}

Fuente: Elaboración propia. 
Los estudios e investigaciones reseñados en la tabla 1 comparten dos problemáticas esenciales:

Problemas en las clasificaciones de los beneficios: En el caso de la clasificación de los beneficios a pesar de que muchos autores introducen algún tipo de clasificación, estas presentan grandes diferencias entre sí (Plessius, Steenbergen y Slot, 2013). Las pautas para clasificar los beneficios de la arquitectura empresarial son muy heterogéneas y no se ha llegado a un consenso al respecto. Esto deriva en la dificultad de realizar un análisis integrador ya que las clasificaciones en la mayoría de los casos no se derivan de un marco teórico (Niemi, 2006; Henk, Marlies y Slot, 2014) y no se diferencian los beneficios en diferentes niveles de abstracción.

No existencia de pruebas empíricas de beneficios: Solo una pequeña parte de los estudios consultados proporciona evidencias objetivas de los beneficios de la arquitectura empresarial (Niemi, 2016). La mayoría de los autores no explican cómo Ilegaron a los beneficios (Tamm, Seddon, Shanks, Reynolds y Frampton, 2011; Plessius, Steenbergen y Slot, 2018), no está claro cómo se obtienen (Niemi y Pekkola, 2019) y se basan solo en evidencia anecdótica (Baharudin Jusuf y Kurnia, 2017). Para Zaher (2017) los beneficios que se han estado publicando en la literatura son muchos y diversos, pero no sostienen evidencias concretas, al depen- der de factores contextuales, la arquitectura empresarial puede conducir a beneficios subjetivos, difíciles de demostrar.

Prevalencia de un enfoque cuantitativo: La mayoría de los estudios sobre beneficios de la arquitectura empresarial han adoptado un enfoque cuantitativo (Niemi, 2016), descuidando el elemento contextual en el que se desarrolla la aplicación de la arquitectura empresarial. Podría parecer positivo que un elevado porcentaje de las investigaciones consultadas propongan escalas cuantitativas, pero en su gran mayoría no se justifican ni se validan correctamente estas escalas.

Diversidad de enfoques: Entre los trabajos consultados existen diferencias en los enfoques para la presentación o análisis de los beneficios de la arquitectura empresarial. Dentro de la diversidad de enfoques se pueden enmarcar las investigaciones consultadas en 5 de estos según se muestra en la tabla 2. El enfoque multidimensional es el más presente con una variada gama de clasificaciones de beneficios que van desde el impacto en los procesos de negocio hasta la utilidad de la arquitectura empresarial en la gestión de la tecnología y la innovación. La generación de valor ha sido un aspecto muy tenido en cuenta sobre todo para cuantificar el impacto real de la arquitectura empresarial, aunque como se explicó anteriormente no ha sido demostrado.

Tabla 2: Descripción del enfoque de los trabajos consultados

\begin{tabular}{|c|c|c|c|}
\hline Enfoque & Explicación & Cantidad & $\%$ \\
\hline Multidimensional & $\begin{array}{l}\text { Se utilizan varios criterios para la evaluación de los beneficios sin enfocar- } \\
\text { se en una dimensión específica. }\end{array}$ & 17 & 54,84 \\
\hline Generación de valor & $\begin{array}{l}\text { Los criterios utilizados para evaluar los beneficios se establecen en base } \\
\text { al valor que la arquitectura empresarial aporta a las organizaciones. }\end{array}$ & 6 & 19,35 \\
\hline $\begin{array}{l}\text { Gobierno digital y } \\
\text { gestión del desem- } \\
\text { peño }\end{array}$ & $\begin{array}{c}\text { Se basa en los beneficios para el gobierno en un contexto digital y en } \\
\text { procesos altamente automatizados. }\end{array}$ & 4 & 12,91 \\
\hline $\begin{array}{l}\text { Cuadro de mando } \\
\text { integral }\end{array}$ & $\begin{array}{l}\text { Se evalúan los beneficios teniendo en cuenta los aportes en cada una de } \\
\text { las dimensiones del cuadro de mando integral. }\end{array}$ & 3 & 9,68 \\
\hline $\begin{array}{l}\text { Gestión del Conoci- } \\
\text { miento }\end{array}$ & $\begin{array}{l}\text { Evalúa los beneficios en base a la generación, tratamiento, almacena- } \\
\text { miento y explotación del conocimiento dentro de las organizaciones. }\end{array}$ & 1 & 3,23 \\
\hline
\end{tabular}

Fuente: Elaboración propia.

Existen investigaciones que exponen resultados concretos sobre el impacto de la implementación de iniciativas de arquitectura empresarial en aspectos como la cultura organizacional y la gestión del cambio a través de la tecnología de la informa- ción (Baharudin y Kurnia, 2017). Algunos de estos trabajos han trazado rutas de éxito a través de las cuales las organizaciones han asumido como parte de sus objetivos principales la realización de cambios en su forma de operar mediante ini- 
ciativas, abarcadoras o parciales, de arquitectura empresarial. Pero estos aportes tienden a la misma carencia de los trabajos de orden académico: no son capaces de aportar evidencia sólida de su existencia, y más aún cuando se trata de elementos subjetivos verificables en el largo plazo como es el caso de la cultura organizacional. En general, la mayoría de los estudios existentes que se centran en los beneficios de la arquitectura empresarial se basan únicamente en la revisión de la literatura disponible (Baharudin, 2017).
Para evaluar la pertinencia de los trabajos consultados en la tabla 1 sobre los beneficios de la arquitectura empresarial se utilizan tres criterios definidos por el autor de la presente investigación: La clasificación coherente de los beneficios, la existencia de un modelo de evaluación y la existencia de evaluación cuantitativa. En la figura 1 analizan las publicaciones analizadas en cuanto al cumplimiento de los tres criterios anteriores.

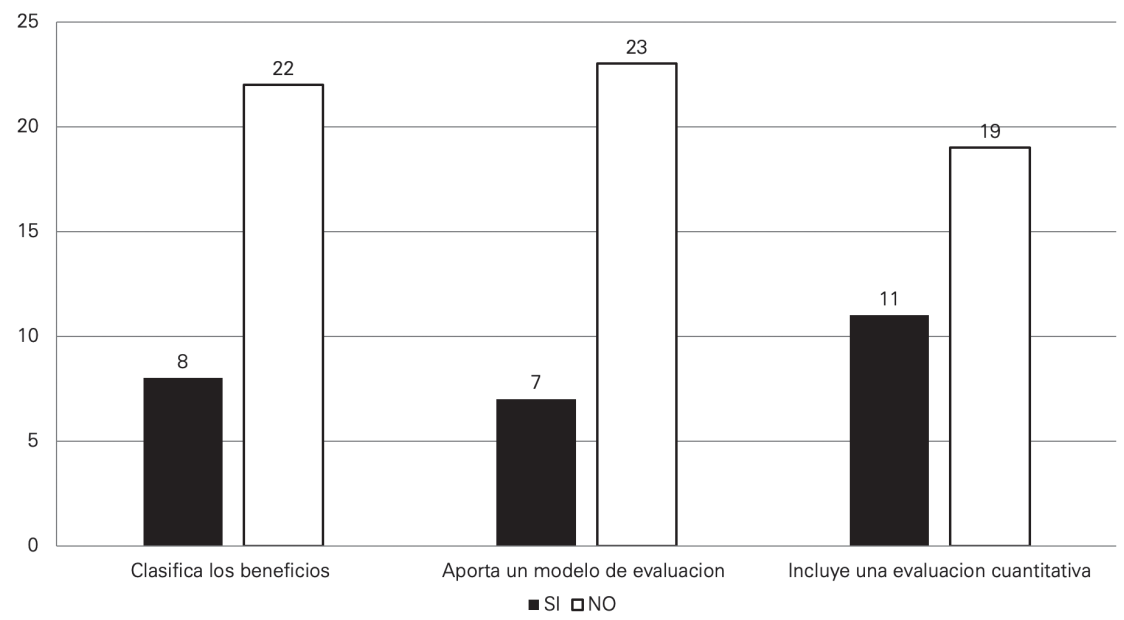

Figura 1: Cumplimiento de los criterios por cada una de las investigaciones. Fuente: Elaboración propia.

\section{Las capacidades organizacionales}

La conceptualización de capacidad organizacional se ha manifestado sobre todo en el ámbito de la dirección estratégica. Según Chavarría (2017) y Dávila (2013) las capacidades organizacionales se consideran aspectos que se construyen socialmente y se orientan a métodos para resolver colectivamente los problemas de las organizaciones y son impactadas por procesos de aprendizaje. Los recursos, junto a las habilidades de la empresa tienen un gran impacto estratégico. Se consideran como un capital para alcanzar los objetivos (Rotundo y Martínez, 2018) y en ocasiones son conocidos como competencias clave (Enginoğlu y Arikan, 2016). Las capacidades organizacionales son complejas combinaciones de activos, personas y procesos que las organizaciones utilizan para transformar los insumos en productos y servicios (Apodaca y cols., 2016). Al poseer una capacidad, la organización desarrolla habilidades para la ejecución de una actividad o proceso según los parámetros planificados y con cierto grado de eficacia
(Winter, 2007; Litchfield y Gentry, 2010). Tanto los recursos con que cuenta una empresa como sus capacidades son cruciales para un buen desempeño, pero los recursos aislados, no son capaces de generar ventajas competitivas (Cardona, 2011). Las capacidades organizacionales también se denominan "competencias distintivas o esenciales", según el término usado por Prahalad y Hammel (1990). Son útiles para crear, ampliar y modificar la base de recursos de la organización (O'Reilly y Tushman, 2011). El enfoque basado en la gestión de las capacidades es considerado como una promesa para la gestión de la tecnología y una de las líneas teóricas de la vista evolucionada de las empresas (Gusberti, Viegas y Echeveste, 2013).

Otros autores como Wang y Zeng (2017) plantean que las capacidades organizaciones pueden ser el fruto del conjunto de las capacidades individuales de una organización que además garantizan los recursos para lograr una ventaja competitiva (Faiz, 2014). En este contexto, un modelo de capacidad empresarial incluye la descripción de capaci- 
dades y conexiones (Bernus y Doumeingts, 2010), describe la arquitectura deseada de funcionalidad (Kangilaski y Shevtshenko, 2017), está destinado a lograr resultados o efectos (Jacobs, von Solms y Grobler y cols., 2016) y describe los requisitos necesarios para llenar los vacíos (Wißotzki, 2016). Las capacidades organizacionales son fundamentales para sustentar las ventajas competitivas de las organizaciones (Lay y Jusoh, 2017) y se han convertido en la base para la implementación de estrategias a largo plazo ya que proveen la dirección básica y son la fuente principal de la rentabilidad para la organización (Espinosa y cols., 2017).

Según Chavarría (2017), existen muchos tipos de clasificaciones de capacidades organizacionales y la definición estandarizada de las mismas no se ha logrado de forma completa. La formulación de capacidades ha quedado a elección libre del investigador 0 autor que aborde el tema, aunque se han establecido algunas tipologías que han sido aceptadas (Wang y Zeng, 2017). Una de estas clasificaciones es la que considera las capacidades dinámicas u operacionales (Winter, 2007; Inan y Bititci, 2015 y Popadiuk y cols., 2018), que define las capacidades operacionales como las rutinas de alto nivel que juntas implementan flujos de procesos que producen salidas. Las capacidades dinámicas construyen, integran y son capaces de reconfigurar las competencias necesarias para adaptarse a los cambios del contexto (Stadler, Helfat y Verona, 2013; Inan y Bititci, 2015; Laaksonen y Peltoniemi, 2018). Muy relacionada con esta clasificación se establecen tres tipos de capacidades: estáticas (primer nivel), dinámicas (segundo nivel) y creativas (tercer nivel). Existen innumerables capacidades organizacionales en muchos campos o áreas de la gestión empresarial. No es objetivo de la presente investigación profundizar en la conceptualización de cada una de las capacidades organizacionales que puedan existir. Para la realización del estudio se eligen cuatro capacidades básicas que según el autor tienen relación con los beneficios de la arquitectura empresarial para las organizaciones: la capacidad de integración de tecnologías de la información, la capacidad de alineación estratégica, la capacidad de gestión de la complejidad y la capacidad de innovación.

Capacidad de integración de tecnologías de la información: Las capacidades tecnológicas son la forma en que las empresas identifican, adoptan, usan, dominan, modifican y crean tecnologías utilizando conocimiento nuevo o existente (Chavarría, 2017). El uso adecuado de capacidades tecnológicas se materializa en los conocimientos y habilidades necesarias para diseñar y crear diferentes productos y servicios (Serna y cols., 2012). La forma en que las compañías apalancan su inversión para crear recursos y habilidades únicas en tecnologías de la información determinan la efectividad de la compañía (García, Díaz y Arias, 2017), estas habilidades organizacionales permiten conectar mediante flujos de información, las actividades de la cadena de suministro con la operación digital del negocio (García y cols., 2017). Según Oliveira y Maçada (2017) las capacidades tecnológicas normalmente se clasifican de tres maneras: capacidades de la infraestructura de tecnologías de la información, capacidades de las personas con las tecnologías de la información y capacidades de gestión de la tecnología. Esta capacidad se enfoca a la habilidad de las organizaciones para integrar las tecnologías de la información a los procesos de negocio de forma sostenible y ágil.

Capacidad de alineación estratégica: La dirección estratégica propone, a partir de la definición de un propósito estratégico, la elaboración de un diagnóstico que incluye un análisis externo e interno. Esta capacidad reivindica el rol que juegan las características internas (recursos) de la organización, como la clave para el establecimiento de las políticas y estrategias para alcanzar ventajas competitivas (Rotundo y Martínez, 2018). El análisis interno se soporta en las directrices que surgen de una perspectiva teórica basada en los recursos (Perdomo, 2003). Por lo tanto, las ventajas competitivas de las empresas tienen su fuente en los recursos, capacidades y aptitudes centrales. Establecer la ventaja competitiva a través del despliegue de los recursos y capacidades, más allá de suponer una protección contra la tormenta competitiva, se ha convertido en el objetivo principal de la estrategia.

Capacidad de gestión de la complejidad: Es muy común verla asociada a competencias clave (Enginoğlu y Arikan, 2016) y se refiere a la posibilidad que tienen las organizaciones para combinar la eficiencia organizacional con la flexibilidad (Schienstock, 2009). Considerada por muchos autores como una capacidad dinámica (Inan, 2016), esta capacidad trata sobre manejar la complejidad a través de la integración de recursos, conocimientos o procesos. La integración es un término en auge en los negocios producto de un incremento constante de la complejidad en la estructura administrativa, los procesos y los sistemas tecnológicos. Esta capacidad en el contexto de la 
investigación se refiere a la habilidad que tiene la organización para integrar procesos, aplicaciones, personas y recursos en sistemas capaces de cumplir las metas previstas y funcionar de forma orgánica.

Capacidad de innovación: Muchos de los estudios sobre los elementos clave que ayudan a las organizaciones a adquirir la capacidad de innovación han propuesto a los recursos y las competencias como elementos fundamentales (Bravo y Herrera, 2009). De acuerdo con la literatura que analiza la gestión del conocimiento, las capacidades dinámicas más comúnmente asociadas a la innovación son: la creación, la absorción, la integración y la reconfiguración del conocimiento. La capacidad de innovación se estima como la característica primordial para descubrir las necesidades de comercialización, hasta convertirse en ventaja competitiva (Asim y Sorooshian, 2019). Desde la perspectiva de los recursos y capacidades, se entiende que detrás de la innovación como proceso empresarial están las capacidades organizacionales. Se toma el significado de esta capacidad tal cual se establece en la bibliografía consultada.

\section{DISCUSIÓN}

Beneficios de la arquitectura empresarial según la capacidad de integración de tecnologías de la información

Dado el gran crecimiento de las organizaciones a nivel de procesos y estructura, cada vez es más difícil alinear negocios y tecnología (Lin y Dyck, 2010). El aumento de la complejidad de los sistemas de información (Theuerkorn, 2005) y su alto costo de mantenimiento, han hecho necesaria una disciplina como la arquitectura empresarial. Muchos de los entornos de procesamiento de información desarrollados en los últimos tiempos han surgido desde dentro de los departamentos comerciales de las organizaciones sin la participación de las unidades de tecnologías de la información (Luisi, 2014). La arquitectura empresarial es capaz de alinear las inversiones en sistemas de información con los objetivos empresariales (Banaeianjahromi, 2018) y tiene el desafío de lograr que estos sistemas coordinen su trabajo y compartan información (Sessions, 2008).

La arquitectura empresarial puede ser útil para controlar los costos (Liimatainen, 2009; Kamoun, 2013; Baharudin Jusuf y Ahsan, 2016 y Halawi y cols., 2018), la eficiencia de la tecnología (Theuerkorn, 2005; Dragstra, 2005 y Proper, Lankhorst, Schönherr, Barjis y Overbeek, 2012;) y los recursos (Baharudin y Kurnia, 2017). Aporta flexibilidad a los sistemas de tecnologías de la información (Abdullah, 2017) en el contexto de la gestión de la cartera de proyectos y en las decisiones de inversión relacionadas con la tecnología (Proper y cols., 2012). Con una arquitectura consistente y bien administrada, la empresa podrá minimizar los riesgos de inversiones equivocadas, contribuir a la entrega de valor de acuerdo con los objetivos comerciales (Perroud y Inversini, 2013) y garantizar que las inversiones apunten a soluciones tecnológicas de vanguardia (Chorafas, 2002). Muchas organizaciones han implementado la arquitectura empresarial para facilitar la transformación digital creando procesos y sistemas ágiles (Lauvrak, Michaelsen y Olsen, 2017). Esta es capaz de alinear las actividades de las tecnologías de la información con los objetivos de la empresa (Perroud y Inversini, 2013) y ofrece sistemas con límites funcionales claros e interfaces que aumentan la flexibilidad de una organización y la alineación de los procesos con las tecnologías emergentes (Rodríguez y Ledo, 2020).

En el campo de la automatización de procesos la arquitectura empresarial puede ser muy útil garantizando que los procesos fluyan mucho más fácilmente entre socios, proveedores, unidades organizativas, sucursales, etc. Garantiza la gestión de la información de acuerdo con las necesidades del negocio con ayuda de sistemas de información y centros almacenamiento de datos (Guerrero, 2016), alcanzando una estandarización en los sistemas de información y una interoperabilidad entre tecnologías. En la práctica, la arquitectura empresarial aumenta las posibilidades de reutilización y reducción de la duplicación de tecnologías de la información (Tamm y cols., 2011), contribuyendo a mejorar la eficacia de las soluciones y a disminuir los tiempos de entrega (Baharudin y Kurnia, 2017). Además, aumenta la interoperabilidad y la integración entre las infraestructuras de tecnologías de la información (Baharudin y Kurnia, 2017) para hacer frente a las tensiones de alineación tradicionales entre empresas y tecnología (Puchol y Collado, 2017).

La arquitectura empresarial contribuye a la disponibilidad oportuna de información (Baharudin Jusuf y Ahsan, 2016) y mejora la disponibilidad y calidad de la información (Tamm y cols., 2011). Para que las organizaciones logren mejorar el rendi- 
miento competitivo es fundamental la alineación o el ajuste de la tecnología con el negocio. De esta alineación salen particularmente beneficiados los sistemas de información ya que la arquitectura empresarial define la estructura adecuada de los mismos (Kotusev, Kurnia, Dilnutt y Taylor, 2020). Un enfoque de arquitectura empresarial para el desarrollo de sistemas de información permite identificar las interrelaciones esenciales entre los componentes que deben alinearse (Baharudin y Ahsan, 2016), garantizando la unificación, intersección e interconexión de los sistemas de información. Las organizaciones a menudo carecen de una visión general clara de sus funciones comerciales, procesos, sistemas de información y plataformas técnicas individuales (Niemi y Pekkola, 2019). La arquitectura empresarial ayuda a reconocer y asimilar el valor de la información externa, las tendencias y los requisitos para transformar los datos en perspectivas comerciales (Zaher, 2017). Muchas organizaciones emplean la arquitectura empresarial como un instrumento para estructurar y administrar sus procesos, sistemas de información e infraestructura técnica desde una perspectiva integral (Henk, vav Marlies y Slot, 2014).

\section{Beneficios de la arquitectura empresarial según la capacidad de alineación estratégica}

Los beneficios estratégicos son efectos positivos que se obtienen a largo plazo y generalmente se ven afectados por una multitud de factores (Niemi y Pekkola, 2019). Tradicionalmente la noción de estrategia juega un papel importante en el discurso de la arquitectura empresarial (Cameron, 2015; Kotusev, Kurnia, Dilnutt y Taylor, 2020). La mayoría de los beneficios entran dentro de esta categoría (Baharudin y Kurnia, 2017). La arquitectura empresarial influye fuertemente en la toma de decisiones de las organizaciones (Plessius, Steenbergen y Slot, 2013). Esto ocurre porque a pesar de que la arquitectura empresarial es en gran medida un fenómeno teórico, puede usarse como una herramienta para poner en práctica la estrategia (Lauvrak, y cols., 2017). De cara al futuro, muchas organizaciones apuestan por la arquitectura empresarial, ya que permite crear una cultura de adaptación y optimización (Bernard, 2012). Para enfrentar mercados dinámicos y cambiantes la arquitectura empresarial aporta agilidad, flexibilidad y capacidad de respuesta para enfrentar cambios y reestructuraciones (Baharudin y Kurnia, 2017).
La arquitectura empresarial permite la identificación del estado actual de la empresa (Plessius, Steenbergen y Slot, 2013) y la describe como un modelo que articula coherentemente todos sus componentes (Arango y Zapata, 2010). Traduce la misión y la visión de la organización en los procesos del negocio y la realización de estos con apoyo de las tecnologías de la información y la infraestructura tecnológica (Silva, 2016). Además, ayuda a comprender el negocio actual y cómo se verá en el futuro, indicando donde se requieren cambios para crear artefactos que se modelen estos cambios (Kangilaski y Shevtshenko, 2017). En la implementación de los cambios, la arquitectura empresarial proporciona conceptos, modelos y herramientas que permiten a las organizaciones enfrentar los desafíos de integrar las áreas estratégicas y los procesos comerciales (Vargas, 2015). Puede desempeñar un potente papel en la configuración de la respuesta de las organizaciones a las condiciones económicas turbulentas (Kamoun, 2013), ya que es una herramienta importante para poner en práctica políticas y estrategias (Halawi y cols., 2018). Esto lo logra proporcionando una hoja de ruta para guiar la organización hacia el logro de su visión y el cumplimiento de las políticas.

La arquitectura empresarial es un paso hacia la implementación de los lineamientos estratégicos (Tamm y cols., 2011) y puede llegar a ser un puente entre la estrategia y la ejecución (Plessius, Steenbergen y Slot, 2013). En la gestión del cambio la arquitectura empresarial ha demostrado ser muy útil. Esta trabaja para lograr una empresa ágil, capaz de evolucionar continuamente dentro de un entorno en constante cambio (Niemi, 2006). La arquitectura empresarial puede ayudar a impulsar el cambio estratégico porque proporciona coherencia en la implementación de la estrategia (Nassiff, 2012) y lo apoya mediante la comunicación y la colaboración (Tamm y cols., 2015). La arquitectura empresarial ayuda a comunicar y a crear un entendimiento compartido (Zaher, 2017) que permite una mejor identificación y gestión de los riesgos (Niemi, 2016) en el contexto de reestructuración (Gabier y cols., 2005). Las organizaciones que aplican la arquitectura empresarial conocen mejor sus objetivos (Tamm y cols., 2011) y están más preparadas para la colaboración entre las partes interesadas (Baharudin y Kurnia, 2017). Al ser el análisis de la estrategia la base del esfuerzo de la arquitectura empresarial (Kotusev y cols., 2020), esta ayuda a traducir los principios, capacidades y objetivos en sistemas y procesos. 
Beneficios de la arquitectura empresarial según la gestión de la complejidad

Uno de los beneficios más importantes de la arquitectura empresarial es su gran potencial para representar de forma holística la organización (Plessius y cols., 2013; Niemi, 2016; Kara y Thakurta, 2018; Hylving y Bygstad, 2018). La arquitectura empresarial ayuda a generar vistas claras y consistentes de la organización (Bernard, 2012), apoya la comprensión colectiva (Lauvrak, y cols., 2017), proporciona soporte en el contexto del cumplimiento normativo (Baharudin y Kurnia, 2017) y se apoya en la gestión de calidad (Proper y cols., 2012).

La arquitectura empresarial integra unidades y dependencias de la empresa (Baharudin y Ahsan, 2016), actuando incluso en aquellos lugares donde la implementación del enfoque a procesos no pudo hacer mucho (Zaher, 2017). Esta integración resuelve la separación entre los grupos de tecnología de la información y los grupos de negocios, la arquitectura empresarial actúa directamente para resolver estas diferencias (Sessions, 2008). Muchos de los problemas de las empresas actuales pueden encontrar soluciones al tener un enfoque definido para gestionar la complejidad (Sessions, 2008; Niemi, 2016). Otros de los elementos que distingue a la arquitectura empresarial es su capacidad de integrar los recursos tecnológicos y procesos comerciales (Theuerkorn, 2005; AbduIlah, 2017), esto permite generar un mayor valor para las empresas, mejorar su rendimiento, su comunicación y su grado de integración (Vargas, 2017). Esta integración además ayuda a alcanzar los objetivos empresariales esenciales de la empresa (Foorthuis, 2012) mediante la integración de personas, procesos, herramientas, recursos, datos e información; para que trabajen de manera sincronizada (Anderson y cols., 2008).

Una arquitectura empresarial relaciona los procesos de trabajo con la infraestructura de tecnologías de la información necesaria para ejecutarlos (Urbaczewski, y Mrdalj, 2018). También hace posible la comunicación sobre diferentes aspectos de la organización y entre sus diferentes dominios (Dragstra, 2005) mediante un lenguaje unificado y estandarizado (Silva, 2016), proporcionando el soporte necesario en el contexto de problemas de diseño organizacional (Proper y cols., 2012). Garantiza la interoperabilidad empresarial (Baharudin y Ahsan, 2016), aumenta la agilidad organizacional (Hylving y Bygstad, 2018) y facilita la solución de problemas de alineación de negocios (Nassiff, 2012). La arquitectura empresarial apoya la evaluación de la transformación empresarial al exponer modelos transparentes de las entidades comerciales y sus complejas interrelaciones (Zaher, 2017).

\section{Beneficios de la arquitectura empresarial según la capacidad de innovación}

Está demostrado que la arquitectura empresarial apoya directamente la innovación empresarial (Nassiff, 2012). Una arquitectura bien definida es un activo importante en el posicionamiento de nuevos desarrollos dentro del contexto de los procesos existentes, sistemas de tecnologías de la información y otros activos de una organización (Lankhorst y cols., 2006). La arquitectura empresarial tiene uno de sus como objetivo claves lograr que la tecnología sea útil a la economía de la innovación ya que los enfoques arquitectónicos y la planificación dinámica ayudan a transformar la empresa radicalmente (Chorafas, 2002). Dentro de la arquitectura de negocios, el modelo de estrategia es responsable de crear negocios innovadores a través de la implementación de múltiples planes de negocios (Abdullah, 2017). En estos planes se tiene en cuenta cómo usar mejor la tecnología y reducir las dependencias de las operaciones humanas (Sessions, 2008), al mismo tiempo que se mejora la innovación y abre nuevas oportunidades (Baharudin y Kurnia, 2017). Una organización que aplique como norma la arquitectura empresarial será siempre más innovadora y abierta al cambio.

La información proporcionada por una arquitectura empresarial es necesaria, por un lado, para determinar las necesidades y prioridades de cambio desde una perspectiva empresarial, y, por otro lado, para evaluar cómo la empresa puede beneficiarse de las innovaciones tecnológicas (Lankhorst y cols., 2006). La arquitectura empresarial también tiene una función de control y ofrece posibilidades de auditoría y diagnóstico (Dragstra, 2005), funciones importantes para proporcionar la base para una futura expansión tecnológica (Halawi y cols., 2018). Los diagnósticos a través de la arquitectura empresarial son abarcadores e integradores. A través de la arquitectura de cualquier organización es factible entender cómo funcionan sus procesos internos y como cada uno puede asumir iniciativas de innovación. 


\section{CONCLUSIONES}

Como toda nueva corriente del conocimiento, los estudiosos de la arquitectura empresarial han tratado con frecuencia de demostrar que es una decisión acertada. Aunque no se equivocan, les ha resultado difícil probar sus argumentos. La comunidad científica a dicho a coro que la arquitectura empresarial es una de las temáticas de la gestión de los negocios con el efecto más integral y abarcador. Aunque es preciso reconocer que es atractiva en su esencia y provoca que sea usada mucho en el ámbito mercadológico, si existe consenso que es necesaria, útil e innovadora. Pero el problema ha estado siempre en demostrarlo y es aquí donde se debe concentrar la comunidad de estudiosos y promotores de la arquitectura empresarial. Es necesario buscar nuevas alternativas, nuevos vínculos y horizontes que permitan promover la arquitectura empresarial como una solución holística a muchos de los problemas actuales que enfrentan empresas, gobiernos y organizaciones de todo tipo. En la presente investigación se logra establecer una nueva vertiente sobre la comprensión y despliegue de beneficios de la arquitectura empresarial: las capacidades organizacionales. Las principales conclusiones de este estudio son dos y se presentan a continuación:

Para demostrar los beneficios de la arquitectura empresarial hay que enfocarse en los criterios de evaluación: La carencia de criterios basados en evidencias prácticas ha dejado muchas dudas en muchos de los estudios. Las iniciativas analizadas en este material comparten la misma inconsistencia al no plantear, al menos basado en evidencia comprobable, sus argumentos. Esto ocurre en gran medida porque los criterios utilizados han sido identificados sin una base teórica que los respalde y no se ajustan concretamente a lo que debe ser un resultado de la arquitectura empresarial. Definir criterios adecuados es el primer paso para una evaluación de beneficios adecuada.

Las capacidades organizacionales se erigen como dimensiones mediadoras entre el impacto la arquitectura empresarial y los resultados de la organización: Enfocar los beneficios de la arquitectura empresarial en base a su impacto en las capacidades organizacionales aporta un vínculo entre las prácticas exitosas de las organizaciones y los esfuerzos realizados en aplicarla. Las 4 capacidades organizacionales escogidas establecen los beneficios de la arquitectura empresarial en términos claros y emula en coherencia con los enfoques tradicionales o las formas habituales de clasificar y presentar los beneficios. Teniendo en consideración los aportes del presente estudio, es muy posible que otros estudios empíricos que puedan realizarse en el futuro tengan mucha más facilidad de demostrar impactos reales en las organizaciones que aplican la arquitectura empresarial. A través de las capacidades organizacionales, estableciendo variables específicas, es posible evaluar la pertinencia de las inversiones en iniciativas de arquitectura empresarial. Los análisis tienen un numero infinito de posibilidades y podrían incluir en el futuro la satisfacción del cliente, las ventas netas, las utilidades y la eficacia de procesos.

\section{BIBLIOGRAFÍA}

Abdallah, A., y Abran, A. (2019). Enterprise architecture measurement: an extended systematic mapping study. I.J. Information Technology and Computer Science, 9, 9-19.

Abdullah, S. (2017). A proposed model to measure the impact of business architecture. Cogent Business \& Management, No 4, 1-8.

Abu Faiz, M. (2014). Organization's core competencies; A key for successful and happy organization. International Journal of Accounting \& Business Management, 2(1), 06-16.

Abunadi, I. (2019). Enterprise architecture best practices in large corporations. Information, 10(293), 1-26.

Aier, S., Fischer, C., y Winter, R. (2011). Construction and evaluation of a meta-model for enterprise architecture design principles. 10 th International Conference on Wirtschaftsinformatik, Zurich, Switzerland.

Aier, S., y Schelp, J. (2010). A reassessment of enterprise architecture implementation. Springer-Verlag Berlin Heidelberg.

Ajer, A. K. S. (2020). Institutional perspective on introducing enterprise architecture. The case of the Norwegian hospital sector [Doctorado, University of Agder]. 
Anderson, M. S., Martin, S. M., Dagli, C., y Miller, A. (2008). Implementing an architectural framework to define and deliver net-centric capability to legacy military air assets operating within a system of systems. IEEE International Systems Conference, Montreal, Canadá,

Apodaca, L. E., Maldonado, S. E., y Máynez, A. I. (2016). La ventaja competitiva, desde la teoría de recursos y capacidades. Revista Internacional Administración \& Finanzas, 9(1), 69-80.

Arango, M. D., y Zapata, J. A. (2010). Arquitectura Empresarial. Una Visión Ceneral. Revista Ingenierías. Universidad de Medellín, Vol. 9 (No 16), 101-111.

Arango Serna, M. D., Londoño Salazar, J. E., y Álvarez Uribe, K. C. (2012). Capacidades de negocio en el contexto empresarial. Revista Virtual Universidad Católica del Norte, 35, 5-27.

Armour, F., y Kaisler, S. (2018). Introduction to the Minitrack on Enterprise Architecture and Business Processes Analysis. Hawaii International Conference on System Sciences, United States of America.

Asim, Z., y Sorooshian, S. (2019). Exploring the role of knowledge, Innovation and technology management (KNIT) Capabilities that influence research and development. Journal of Open Innovation, 5(21), 1-47.

Azizi, L., y Sumitra, I. D. (2019). Designing of enterprise architecture for interior furniture production based on TOGAF 9.1. IOP Conference Series: Materials Science and Engineering.

Baena Paz, G. (2017). Metodología de la investigación serie integral por competencias. Grupo Editorial Patria.

Baharudin Jusuf, M., y Ahsan, K. (2016). Role of enterprise architecture in healthcare organizations and knowledge-based medical diagnosis system. JISTEM, Journal of Information Systems and Technology Management, 13(2), 1807-1775.

Banaeianjahromi, N. (2018). On the role of enterprise architecture in enterprise integration [Doctorado, Lappeenranta University of Technology].

Bernard, S. A. (2012). An Introduction to Enterprise Architecture (Third Edition). AuthorHouseTM.
Bernus, P., y Doumeingts, G. (2010). Enterprise Architecture, Integration and Interoperability. Springer, Brisbane, Australia.

Bravo, E., y Herrera, L. (2009). Generación de capacidades dinámicas mediante la innovación organizacional: Un múltiple estudio de casos exploratorio. XIII Congreso de Ingeniería de Organización, Barcelona, España.

Cameron, B. H. (2015). The need for enterprise architecture for enterprise-wide big data. A Journal of Law And Policy, 10 (3), 827-863.

Cardona, R. A. (2011). Estrategia basada en los recursos y capacidades. Criterios de evaluación y el proceso de desarrollo. Revista Electrónica Fórum Doctoral, 4, 113-147.

Caruso, M. J. (2019). Strategies to improve adoption of the Federal Enterprise Architecture Framework [Doctorado, Walden University].

Chakraborty, P., y Sarkar, A. (2017). Context Driven Approach for Enterprise Architecture Framework. LNCS, 10244, 277-289.

Chavarría, J. H. (2017). Capacidades tecnológicas y organizacionales de las empresas mexicanas participantes en la cadena de valor de la industria aeronáutica. Economía Teoría y Práctica, 47, 65-98.

Chávez Rodríguez, M. M., y Villar Ledo, L. (2020). Marco de Procesos de Negocio de Telecomunicaciones. Análisis de beneficios y evaluación de viabilidad. Tono, 16 (1), 65-75.

Chorafas, D. N. (2002). Enterprise architecture and new generation information systems. CRC Press LLC.

Cretu, L. G. (2014). Designing enterprise architecture frameworks. Integrating business processes with IT infrastructure. Taylor \& Francis, Apple Academic Press.

Dang, D. D., y Pekkola, S. (2017). Systematic literature review on enterprise architecture in the public sector. The Electronic Journal of E-Government, 15(2), 132-154.

Dávila, J. C. (2013). Capacidades organizacionales: dinámicas por naturaleza. Cuadernos de Administración, 26(47), 11-33.

Díaz Herrera, C. (2018). Investigación cualitativa y análisis de contenido temático. Orientación intelectual de revista Universum. Revista General de Información y Documentación, 28(1), 119-142. 
Dragstra, P. (2005). Enterprise architecture the selection process of an Enterprise Architecture Toolset to support understanding and governing the enterprise [Maestría, Eindhoven University of Technology].

Enginoğlu, D., y Arikan, C. L. (2016). A literature review on core competencies. International Journal of Management (IJM), 7(3), 120-127.

Erdenebold, T., Rho, J. J., y Hwang, Y. M. (2019). Blockchain reference model and use case for supply chains within enterprise architecture. Journal of Information Technology and Architecture, 16(1), 1-10.

Farazmand, E., y Moeini, A. (2011). A framework for knowledge management architecture. 13th International Conference on Enterprise Information Systems, 425-430.

Ferreira de Santana, A. T. (2017). Enterprise architecture analysis based on network paradigm: a framework proposal and empirical evaluation [Doctorado, Universidade Federal de Pernambuco].

Fischer, C., Winter, R., y Aier, S. (2010). What Is an enterprise architecture principle? Towards a consolidated definition. Computer and Information Science 2010, 193-205.

Foorthuis, R. (2012). Project compliance with enterprise architecture [Doctorado, Utrecht University, Utrecht].

Fratila, L. A. (2020). Enterprise architecture and corporate governance, a cohesive approach towards cloud migration in the banking industry. International Journal of Economics, Commerce and Management, VIII (5).

Gabier, B., Seymour, L. F., y Van Belle, J.-P. (2005). Benefits and Factors Driving Enterprise Architecture Development in a Large South African Utility Company. University of Cape Town.

Gampfer, F. (2019). Investigation on the future of enterprise architecture in dynamic environments [Doctorado, Universidad de Murcia].

García, A. A., Díaz, H. E., y Arias Pérez, J. E. (2017). Capacidades de tecnologías de información y capacidades de negocio electrónico (e-business): efecto mediador de la capacidad de absorción. Información Tecnológica, 28(1), 47-64.
Gayatri, P., y Nagalakshmi, V. (2019). Benefits of government enterprise architecture: context of certain ea initiatives in India. International Journal of Engineering Research and Technology. 12(5), 692-696.

Giachetti, R. E. (2015). Evaluation of the DoDAF Meta-Model's Support of Systems Engineering. Procedia Computer Science, 4, 254-260.

Guerrero Silva, W. G. (2016, enero). Arquitectura empresarial. Dominios y beneficios. Revista de la Facultad de las Ciencias Económicas y Empresariales, Volumen 16 (1), 87-92.

Guix Oliver, J. (2008). El análisis de contenidos: ¿qué nos están diciendo? Revista Calidad Asistencial, 23(1), 26-30.

Gusberti, T. D. H., Viegas, C., y Echeveste, M. E. S. (2013). Organizational capability deployment analysis for technology conversion into processes, products and services. Journal of Technology Management an Innovation, 8(4), 129-142.

Halawi, L., McCarthy, R., y Farah, J. (2018). Where We are with Enterprise Architecture. 4801.

Hernández Sampieri, R., Fernández Collado, C., y Baptista Lucio, M. del P. (2014). Metodología de la Investigación. McGRAW-HILL.

Henk, P., vav Marlies, S., y Slot, R. (2014). Perceived benefits from enterprise architecture. Eighth Mediterranean Conference on Information Systems, Verona. Italia.

Hylving, L., y Bygstad, B. (2018). Responding to Enterprise Architecture Initiatives: Loyalty, Voice and Exit. Hawaii International Conference on System Sciences, 2363-2372.

Inan, G. G., y Bititci, U. S. (2015). Understanding organizational capabilities and dynamic capabilities in the context of micro enterprises: a research agenda. Procedia, Social and Behavioral Sciences, 210(15), 310-319.

Jacobs, P. C., von Solms, S. H., y Grobler, M. M. (2016). Towards a framework for the development of business cybersecurity capabilities. The Business and Management Review, 4(7), 51-61.

Jayakrishnan, M., Mohamad, A. K., y Abdullah, A. (2019). Enterprise Architecture Embrace Digital Technology in Malaysian Transportation Industry. International Journal of Engineering and Advanced Technology, 8(4), 852-859. 
Kamoun, F. (2013). Rethinking the role of enterprise architecture during times of economic downturn: a dynamic capabilities approach. Journal of Information Technology Management, XXIV (1), 26-48.

Kangilaski, T., y Shevtshenko, E. (2017). Do we need capabilities in our management system? Journal of Machine Engineering, 17(1), 88-100.

Kar, S., y Thakurta, R. (2018). Planning for Digital Transformation: Implications for Institutional Enterprise Architecture. Twenty-Second Pacific Asia Conference on Information Systems, Japan.

Kotusev, S. (2016). Enterprise Architecture Frameworks: The Fad of the Century. British Computer Society (BCS).

Kotusev, S. (2018). TOCAF-based Enterprise Architecture Practice: An Exploratory Case Study. Communications of the Association for Information Systems, 43(20).

Kotusev, S. (2019). Fake and Real Tools for Enterprise Architecture: The Zachman Framework and Business Capability Model. Enterprise Architecture Professional Journal (EAPJ).

Kotusev, S., Kurnia, S., Dilnutt, R., y Taylor, P. (2020). Can Enterprise Architecture Be Based on the Business Strategy? Hawaii International Conference on System Sciences, 56135622.

Kotusev, S., Singh, M., y Storey, I. (2015). Consolidating Enterprise Architecture Management Research. 48th Hawaii International Conference on System Sciences, 4069-4078.

Laaksonen, O., y Peltoniemi, M. (2018). The Essence of Dynamic Capabilities and their Measurement. International Journal of Management Reviews, 20(1), 184-205.

Lange, M., Mendling, J., y Recker, R. (2012). Realizing benefits from enterprise architecture: a measurement model. ECIS 2012.

Lankhorst, M. (2009). The Enterprise Engineering Series. Modelling, Communication and Analysis (Second edition). Springer.

Lankhorst, M. M., Jonkers, H., ter Doest, H. W. L., Arbab, F., Bosma, H., y Wieringa, R. J. (2006). Enterprise architecture: Management tool and blueprint for the organisation. Information Systems Front, 8, 63-66.
Laschitza, J., y Undén, M. (2017). Enterprise Architecture Implementation [Maestría, University of Cothenburg].

Lauvrak, S., Michaelsen, V. M., y Olsen, D. H. (2017). Benefits and Challenges in Enterprise Architecture Management: A Case Study of The Norwegian Labour and Welfare Administration. Bibsys Open Journal Systems, 25(1).

Lay, T. A., y Jusoh, R. (2017). Organizational capabilities, strategic management accounting and firm performance. Jurnal Akuntansi Dan Keuangan Indonesia, 14(2), 222-246.

Lê, L. (2020). Diagramming Multi-Level Service-Oriented Enterprise Architecture. SN Computer Science, 1(14), 1-25.

Lin, F., y Dyck, H. (2010). The Value of Implementing Enterprise Architecture in Organizations. International Information Management Association, Inc.

Litchfield, R. C., y Gentry, R. J. (2010). Perspective-taking as an organizational capability. Strategic Organization, 8(1), 187-205.

Llanos Cuenca, A. B., \& Ortiz, A. (2011). An enterprise engineering approach for the alignment of business and information technology strategy. International Journal of Computer Integrated Manufacturing. 24(11), 974-992.

Londoño Salazar, J. E. (2014). Modelo funcional de Integración de la Arquitectura Empresarial de 'N' entidades alrededor de un grupo empresarial. Un enfoque de orientación a servicios y modelado de capacidades de negocio [Doctorado, Universidad Nacional de Colombia Facultad de Minas].

Luisi, J. V. (2014). Pragmatic Enterprise Architecture. Strategies to Transform Information Systems in the Era of Big Da. Elsevier Inc.

Lukhele, C. F., Ngassam, E. K., y Osunmakinde, I. (2015). A Framework for Measuring the Value of Enterprise Architecture in South African Telecommunications Organizations. LNICST 147, 282 - 292.

Marini, G. (2019). Enterprise Architecture and Digital Transformation [Maestría, NOVA-School of Business and Economics].

Minoli, D. (2008). Enterprise Architecture A to Z. Frameworks, Business Process Modeling, SOA, and Infrastructure Technology. Auerbach Publications. Taylor \& Francis Group, LLC. 
Moscoso-Zea, O., Paredes Gualtor, J., y Luján-Mora, S. (2019). Enterprise Architecture, an enabler of change and knowledge management. Enfoque UTE, 10(1).

Morán Delgado, G., y Alvarado Cervantes, D. G. (2010). Métodos de investigación. Pearson Custom Publishing.

Nassiff, E. (2012). Understanding the Value of Enterprise Architecture for Organizations: A Grounded Theory Approach [Maestría, Nova Southeastern University].

Niemann, K. D. (2005). From Enterprise Architecture to IT Governance. Friedr. Vieweg \& Sohn Verlag, GWV Fachverlage GmbH.

Niemi, E. (2006). Enterprise Architecture Benefits: Perceptions from Literature and Practice. IBIMA Conference Internet \& Information Systems in the Digital Age, Italy.

Niemi, E. (2016). Enterprise Architecture Benefit Realization. Tampere University of Technology.

Niemi, E. I., y Pekkola, S. (2016). Enterprise Architecture Benefit Realization: Review of the Models and a Case Study of a Public Organization. The DATA BASE for Advances in Information Systems, 47(3), 55-80.

Niemi, E., y Pekkola, S. (2019). The Benefits of Enterprise Architecture in Organizational Transformation.

Nilsen, O. R. (2012). Enterprise Content Management: an analysis of contemporary practice and its relationships with Enterprise Architecture [Maestría, University of Agder].

Noran, O. (2018). An analysis of the Zachman Framework for Enterprise Architecture from the GERAM perspective. IFAC Annual Reviews in Control, 27(2), 163-183.

Nygård, M., \& Olsen, D. H. (2016). Enterprise architecture implementation challenges: an exploratory study of the Norwegian health sector. Bibsys Open Journal Systems, 24(1), 28-30.

Okhrimenko, A. (2017). Comparing Enterprise Architecture Frameworks-A Case Study at the Estonian Rescue Board [Maestría, University of Tartu. Institute Of Computer Science].

Oliveira, D. de L., y Maçada, A. C. (2017). IT capabilities' business value: analysis of multi-level performance in Brazilian organizations. Cestión de la Producción, 24(2), 295-309.
Open Group. (2011). TOGAF Version 9.1 A Pocket Guide.

Orabona, A. E. (2016). Building a foundation for the implementation of an enterprise architecture for the Argentinian Army [Maestría, Naval Postgraduate School].

O'Reilly, C. A., y Tushman, M. L. (2011). Organizational Ambidexterity in Action: How Managers explore and exploit. California Management Review, 53(4), 1-18.

Pähler, P., Morency, M. C., y Lapalme, J. (2019). Examination of explicit definitions of enterprise architecture. International Journal of Engineering Business Management, 11, 1-18.

Pasaribu, F. A., Sipahutar, J. H., y Situmorang, B. P. (2019). Designing Enterprise Architecture in Hospitals Group.

Perdomo Ortiz, J. (2003). La arquitectura organizacional y las capacidades estratégicas. INNOVAR, revista de ciencias administrativas y sociales, No. 22, 55-62.

Perroud, T., y Inversini, R. (2013). Enterprise Architecture Patterns. Practical Solutions for Recurring IT-Architecture Problems. Springer-Verlag Berlin Heidelberg.

Plessius, H., Steenbergen, M. van, y Slot, R. (2018). The enterprise architecture value framework. Twenty-Sixth European Conference on Information Systems, United Kindong.

Plessius, H., van Steenbergen, M., y Slot, R. (2013). Towards an Enterprise Architecture Benefits Measurement Instrument.

Ponce Espinosa, G., Espinoza, D. L., Ríos Zaruma, J., y Tapia C, K. G. (2017). Capacidades organizacionales generadoras de valor: análisis del sector industrial. Revista de Ciencias de la Administración y Economía|, VII (13), 143-162.

Popadiuk, S., Souza Luz, A. R., y Kretschmer, C. (2018). Dynamic Capabilities and Ambidexterity: How are These Concepts Related? RAC, 22(5), 639-660.

Prahalad, C. y Hammel, G. (1990). The core competence of the corporation. Harvard Business Review,1-15.

Priyanto, A. A. (2013). Bringing Bringing Enterprise Architecture to the Boardroom [Maestría, School of Management and Governance. University of Twente]. 
Proper, E., Lankhorst, M. M., Schönherr, M., Barjis, J., y Overbeek, S. (2012). Trends in Enterprise Architecture Research. Springer-Verlag.

Qurratuaini, H. (2017). Designing enterprise architecture based on TOGAF 9.1 framework. IOP Conference Series: Materials Science and Engineering. International Conference on Engineering and Applied Technology (ICEAT), United States of America.

Randall, N. (2018). Enterprise Architecture Content Strategy.

Roach, T. (2011). CAPSICUM. A Semantic framework for Strategically Aligned Business Architecture. UNSW. School of Information Systems and Technology Management.

Rogier, P. F., van de Wetering, R., y Joosten, S. (2020). Enterprise architecture alignment.

Ross, J. W., Weill, P., y Robertson, D. C. (2006). Enterprise Architecture as Strategy. Creating a Foundation for Business Execution. Harvard Business School Press.

Ross, J. W., Weill, P., y Robertson, D. C. (2014). Enterprise Architecture as Strategy.

Rotundo, G. J. Z., y Martínez, A. M. (2018). Capacidades Dinámicas de la Organización: Revisión de la Literatura y un Modelo Propuesto. Investigación Administrativa, 47(121).

Rouhani, B. D., y Nikpay, F. (2012). Agent-Oriented Enterprise Architecture: new approach for Enterprise Architecture. International Journal of Computer Science Issues, 9(6), 331-334.

Saha, P. (2012). Enterprise Architecture as Platform for Connected Government. Understanding the Impact of Enterprise Architecture on Connected Government A Qualitative Analysis. NUS Institute of Systems Science.

Saint-Louis, P., y Lapalme, J. (2018). An exploration of the many ways to approach the discipline of enterprise architecture. International Journal of Engineering Business Management, 10, 1-26.

Sanchez Puchol, F., y Pastor Collado, J. A. (2017). A First Literature Review on Enterprise Reference Architecture. The 11th Mediterranean Conference on Information Systems (MCIS), Genoa, Italy.
Sari, T. R., Rahmawati, E., y Harafani, H. (2019). TOCAF ADM to Improve the Promotion of Farm Edu-Tourism in Pondok Rangon Area. Journal Publications \& Informatics Engineering Research, 3(2).

Schekkerman, J. (2011). Enterprise Architecture Tool Selection Guide. Institute For Enterprise Architecture Developments (IFEAD).

Schienstock, G. (2009). Organizational Capabilities: Some reflections on the concept. University of Tampere.

Sessions, R. (2008). Simple Achitectures for Complex Enterprises. Microsoft Press.

Sessions, R. (2011). Revamping Public Sector IT Procurement to Favour Success and Small Business. Consortium for Untangling Enterprise Complexity.

Sessions, R., y deVadoss, J. (2014). A Comparison of the Top Four Enterprise Architecture Approaches in 2014.

Sidorova, A., y Kappelman, L. (2010). Realizing the Benefits of Enterprise Architecture: An Actor-Network Theory Perspective. College of Business, University of North Texas.

Silva Rodriguez, L., y Amaral, L. (2010). Multidimensional value of enterprise architectures. IADIS International Conference Information Systems (IADIS'2010). United States of America.

Simon, D., Fischbach, K., y Schoder, D. (2013). An Exploration of Enterprise Architecture Research. Communications of the Association for Information Systems, 32(1).

Sofyana, L., y Putera, A. (2019). Business architecture planning with TOGAF framework. IOP Publishing.

Stadler, C., Helfat, C. E., y Verona, G. (2013). The Impact of Dynamic Capabilities on Resource Access and Development. Organization Science, 24(6), 1782-1804.

Stroud, R. O. (2019). Complexity Frameworks in Enterprise Design [Doctorado, Texas Tech University].

Sukur, A. (2020). Enterprise architecture routinization and use of enterprise architecture standards to achieve information technology flexibility and enterprise agility [Doctorado, Capella University]. 
Sultanow, E., Schroeder, K., Brockmann, C., y Cox, S. (2016). A Multidimensional Classification of 55 Enterprise Architecture Frameworks. Twenty-second Americas Conference on Information Systems, San Diego. EEUU.

Sureda, J., Oliver, M. F., Comas, R., y Guerrero, R. M. (2010). Fuentes de información bibliográfica a través de Internet para investigadores en educación. REDINED.

Tamm, T., Seddon, P. B., Shanks, G., Reynolds, P., y Frampton, K. M. (2015). How an Australian Retailer Enabled Business Transformation Through Enterprise Architecture. MIS Quarterly Executive, 14(1), 181-193.

The Open Group. (2018). The TOGAF Standard, Version 9.2.

Theuerkorn, F. (2005). Lightweight enterprise architectures. CRC Press LLC.

Tome, P., y Costa, E. (2010). TREEAD A Tool that Enables the Re-use of Experience in Enterprise Architecture Description. 332-343.

Urbaczewski, L., y Mrdalj, S. (2018). A Comparison of Enterprise Architecture Frameworks. Issues in Information Systems, Volume VII (2), 18-23.

Vargas, A. (2015). Propuesta de Arquitectura Empresarial en el Contexto de Colaboración Jerárquica para el Soporte a la Toma de Decisiones en Situaciones de Eventos Inesperados. [Doctorado, Universitat Politècnica de València].

Vargas, N. (2017). Definición de un marco de referencia de arquitectura empresarial para PYMEs. Iteraciones de capacidad y desarrollo arquitectónico ADM TOGAF. [Pregrado, Universidad Tecnica Particular de Loja].

Vermeulen, R. J. M. (2013). Design of an Enterprise Architecture for information requirements identification: An exploratory study within the high-tech industry. TUE. School of Industrial Engineering.

Wang, X., y Zeng, Y. (2017). Organizational Capability Model: Toward Improving Organizational Performance. Journal of Integrated Design and Process Science, 21(1).
Werewka, J. (2018). Developing Conformance Between Project Management and Enterprise Architecture Governance on the Basis of a PMBOK Case. Advances in Intelligent Systems and Computing. 38th International Conference on Information Systems Architecture and Technology-ISAT 2017, Poland.

Winter, K., Buckl, S., Matt hes, F., y Schweda, C. M. (2010). Investigating the state-of-the-art in enterprise architecture management methods in literature and practice. 90. Mediterranean Conference of Information Systems, Germany.

Winter, R., y Fischer, R. (2007). Essential layers, artifacts, and dependencies of enterprise architecture. Journal of Enterprise Architecture.

Winter, S. G. (2007). Dynamic Capability as a Source of Change. Oxford University Press.

Wißotzki, M. (2016). Method support for enterprise architecture management capabilities [Doctorado, Faculty of Computer Science and Electrical Engineering at the University of Rostock].

Zachman, J. A. (1987). A framework for information systems architecture. IBM Systems Journal, 26(3), 454-470.

Zachman, John A. (2011). The practice of enterprise architecture. Zachman International.

Zaher, A. M. (2017). The Enterprise Architecture: An Empirical Study on the Organisational Benefits and Success Factors [Maestría, the Business School University of Roehampton London]. 
Revista Academia a Negocios Vol.7 (1) 2021 pp. 1-14 